\title{
Survival-associated alternative splicing signatures in soft tissue sarcomas
}

\author{
Xianghai Ren ${ }^{1,2}$, Qi Jiang ${ }^{3}$, Long Li $^{1,2}$ \\ ${ }^{1}$ Department of Pediatric Surgery, Capital Institute of Pediatrics, Beijing 100020, China; ${ }^{2}$ Graduate School of Peking Union Medical College, \\ Chinese Academy of Medical Sciences, Beijing 100730, China; ${ }^{3}$ Department of Pathology and Pathophysiology, Hubei Provincial Key Laboratory of \\ Developmentally Originated Disease, School of Basic Medical Sciences, Wuhan University, Wuhan 430071, China \\ Correspondence to: Long Li, MD, PhD. Department of Pediatric Surgery, Capital Institute of Pediatrics, No.2 Yabao Road, Chaoyang District, Beijing \\ 100020, China. Email: lilong23@126.com. \\ Provenance and Peer Review: This article was commissioned by the Editorial Office, Annals of Translational Medicine. The article did not undergo \\ external peer review. \\ Comment on: Hong W, Zhang W, Guan R, et al. Genome-wide profling of prognosis-related alternative splicing signatures in sarcoma. Ann Transl \\ Med 2019;7:557.
}

Submitted Dec 30, 2019. Accepted for publication Jan 16, 2020.

doi: 10.21037/atm.2020.01.113

View this article at: http://dx.doi.org/10.21037/atm.2020.01.113

Sarcomas, which are a group of connective tissue tumors, are typically categorized into two broad groups: softtissue sarcomas and bone sarcomas. According to their morphological and molecular genetic features, they can be further classified as more than 70 subtypes with variable pathologic features and clinical presentations (1). Despite the diversity in classification, sarcomas account for only about $1-2 \%$ of tumors in adults, $10 \%$ in adolescents and young adults (15-29 years old), and $6-15 \%$ in pediatric patients ( $<15$ years old) (2). Precisely because of their rarity and marked heterogeneity, sarcomas are often misdiagnosed and misrecognized initially. Furthermore, the specific pathogenesis of many sarcoma types remains largely unclear. With the advances in chemotherapy, surgery, and systemic therapy over the past four decades, the overall and event-free survival of patients with certain sarcoma types including Ewing sarcoma, rhabdomyosarcoma, and osteosarcoma has improved significantly (3-5). Nevertheless, the survival outcomes for other sarcomas, such as synovial sarcoma and alveolar soft part sarcoma remain barely satisfactory (6). Emerging research on the pathogenesis of sarcomas therefore holds promise for finding new therapies and further improving overall survival and quality of life.

As an important form of genetic regulation in eukaryotes, alternative splicing (AS) is a process whereby multiple transcripts of different functions are encoded from a single gene by selectively removing or retaining exons and/or introns from the precursor RNA (7). It is estimated that AS events occur in over $90 \%$ of human genes, and they play a crucial role in increasing proteomic and transcriptomic diversity. Similarly, abnormal variations in AS may also widely participate in the biogenesis and progression of cancer and may arise from mutations in the splicing regulatory elements of specific tumor genes or from changes in splicing regulatory mechanisms (8). Additionally, the dysregulated splicing behavior was determined to be related to abnormal splicing factor expression (9). The study of abnormal AS events in cancer is a meaningful research approach that not only offers a rich source of novel molecular biomarkers for early diagnosis and represents potential targets for oncotherapy, but it also provides ideal markers for predicting tumor prognosis.

Splicing pattern dysregulation is demonstrated to affect the progression of some types of sarcomas. Previous studies have reported that the AS of MEF2C and ARID1A play important roles in the pathogenesis of rhabdomyosarcoma and Ewing sarcoma, respectively $(10,11)$. However, these studies were limited to detecting AS events or specific genes associated with a particular type of sarcoma. Cancer is the result of multiple mechanisms acting together. Besides, different sarcoma subtypes may show various AS events, which in turn could help to identify the pathological subtypes of sarcomas (12). Therefore, integrating genomewide splicing events in sarcomas into an aggregated model 
for comprehensive analysis is meaningful and necessary. The Cancer Genome Atlas (TCGA) contains RNA-seq data and relevant clinical information for a relatively large number of patients with soft-tissue sarcoma, which provides the unique opportunity to systematically study the relationship between abnormal splicing events and the survival outcomes of patients with sarcoma.

In Annals of Translational Medicine, Hong et al. (13) attempted to address this issue by characterizing the genome-wide AS events of 261 patients with soft-tissue sarcoma in TCGA. Of these 261 patients, the cumulative 5 -year overall survival rate was $54.7 \%$, and disease-free survival rate was $40.3 \%$. The median age at diagnosis was 61.4 years (20.6-90.0 years), the gender distribution was roughly equal (M:F $=0.84: 1$ ) and the primary site in most patients was in the retroperitoneum and peritoneum (100 cases, $38.3 \%$ ). The distribution of tumor histology types had no apparent trend, and the leiomyosarcoma type (105 cases, $40.2 \%$ ) accounted for the largest proportion of all patients. In this study, 3,610 total events were found to be prognosis-related. Based on these survival-related AS events, the comprehensive prognostic predictor, which integrated the six types of AS events except for the mutually exclusive exons, was tested to have the greatest performance in prognosis prediction. Another recent study that analyzed a soft tissue sarcoma cohort from TCGA (206 patients) also showed that the prognostic model of AS events had great potential in clinical practice (14). Additionally, Hong et al. attempted to investigate the potential molecular mechanism associated with poor prognosis in soft-tissue sarcomas, of which the focal adhesion pathway was found to be the most critical. Focal adhesion pathways (particularly Src family kinases and integrins), which play important roles in tumor escape, metastasis, proliferation, and drug resistance, can be regarded as promising therapeutic targets for sarcomas (15). However, these preliminary hypotheses require further verification.

Splicing factors, which belong to a group of large, complex, and highly dynamic ribonucleoproteins, are involved in the formation of splicing complexes and execute splicing events (16). Mutations in some core splicing components were found to participate in the occurrence of some tumors and other diseases. SRSF1 was the first splicing factor found to play roles in cancer via promoting cell transformation by regulating AS of MNK2 (17). Furthermore, it has been demonstrated that some splicing factors, such as hnRNP A1 and hnRNP A2, can regulate thousands of AS events and are overexpressed in many human cancer types $(18,19)$. In the context of cancer, it is highly possible that survivalrelated splicing factors are related to cancer biogenesis and progression. Hence, identification of these putative factors and the corresponding up/downstream targets provides the opportunity to identify numerous novel therapeutic approaches. In addition, splicing factor genes are reported to generally exhibit dysregulated expression in cancer (9). However, among different tumor types and even subtypes, the expression of splicing factor genes varies substantially. In soft tissue sarcomas (excepting synovial sarcoma), only a few genes (ATRX, RB1, and TP53) are highly recurrently mutated across sarcoma types (20), compared with $71 \%$ of the genes that are significantly differentially expressed in breast cancer (9). This suggests that the patterns of dysregulated gene expression targeted at splicing factors among cancer types vary significantly. Of 71 total splicing factors, Hong et al. screened out 26 that were survivalrelated and found that most were positively correlated with risk survival prognostic AS events (13). They also identified 14 driver genes in 261 sarcoma patients (13). Among these driver genes, mutations in TP53, RB1, and ATRX were most common, which was similar to the findings of a previous study (20). Conceivably, the dysregulated gene expression and multiple splicing factors have a particular impact on sarcomas survival by regulating AS events. Further functional studies are needed to confirm the true relevance of these genes and factors in AS and carcinogenesis.

Although it may be more appropriate to analyze the AS events in different sarcoma subtypes separately (at least in a few subtypes that contain relatively more patients) because sarcomas are a heterogeneous group of tumors containing diverse biological behaviors, the authors should be commended for undertaking the first research on AS events in soft-tissue sarcomas at the genome-wide level. They constructed the novel field of prognosis prediction models, identified splicing factors and driver genes that are related to poor prognosis, and paved the way for further research regarding potential molecular-targeted therapies in soft-tissue sarcomas. More attention should be paid to future experimental verification of the potential molecular interactions and detailed regulatory networks of the AS process. Besides, relative to other cancers, sarcomas disproportionately affect the young and the current study failed to focus on bone sarcomas; we want to encourage AS research to focus on bone sarcomas and young sarcoma patients to improve the level of diagnosis and treatment of these diseases. 


\section{Acknowledgments}

Funding: None.

\section{Footnote}

Conflicts of Interest: The authors have no conflicts of interest to declare.

Ethical Statement: The authors are accountable for all aspects of the work in ensuring that questions related to the accuracy or integrity of any part of the work are appropriately investigated and resolved.

Open Access Statement: This is an Open Access article distributed in accordance with the Creative Commons Attribution-NonCommercial-NoDerivs 4.0 International License (CC BY-NC-ND 4.0), which permits the noncommercial replication and distribution of the article with the strict proviso that no changes or edits are made and the original work is properly cited (including links to both the formal publication through the relevant DOI and the license). See: https://creativecommons.org/licenses/by-nc-nd/4.0/.

\section{References}

1. Tawbi HA, Burgess M, Bolejack V, et al. Pembrolizumab in advanced soft-tissue sarcoma and bone sarcoma (SARC028): A multicentre, two-cohort, single-arm, openlabel, phase 2 trial. Lancet Oncol 2017;18:1493-501.

2. van der Graaf WTA, Orbach D, Judson IR, et al. Soft tissue sarcomas in adolescents and young adults: a comparison with their paediatric and adult counterparts. Lancet Oncol 2017;18:e166-75.

3. Spraker-Perlman HL, Barkauskas DA, Krailo MD, et al. Factors influencing survival after recurrence in osteosarcoma: A report from the Children's Oncology Group. Pediatr Blood Cancer 2019;66:e27444.

4. Kreyer J, Ranft A, Timmermann B, et al. Impact of the Interdisciplinary Tumor Board of the Cooperative Ewing Sarcoma Study Group on local therapy and overall survival of Ewing sarcoma patients after induction therapy. Pediatr Blood Cancer 2018;65:e27384.

5. Guérin F, Rogers T, Minard-Colin V, et al. Outcome of localized liver-bile duct rhabdomyosarcoma according to local therapy: A report from the European Paediatric SoftTissue Sarcoma Study Group (EpSSG)-RMS 2005 study. Pediatr Blood Cancer 2019;66:e27725.

6. Lewin J, Puri A, Quek R, et al. Management of sarcoma in the Asia-Pacific region: resource-stratified guidelines.
Lancet Oncol 2013;14 e562-70.

7. Bush SJ, Chen L, Tovar-Corona JM, et al. Alternative splicing and the evolution of phenotypic novelty. Philos Trans R Soc Lond B Biol Sci 2017. doi: 10.1098/ retb.2015.0474.

8. Climente-González H, Porta-Pardo E, Godzik A, et al. The Functional Impact of Alternative Splicing in Cancer. Cell Rep 2017;20:2215-26.

9. Sveen A, Kilpinen S, Ruusulehto A, et al. Aberrant RNA splicing in cancer; expression changes and driver mutations of splicing factor genes. Oncogene 2016;35:2413-27.

10. Zhang M, Zhu B, Davie J. Alternative splicing of MEF2C pre-mRNA controls its activity in normal myogenesis and promotes tumorigenicity in rhabdomyosarcoma cells. J Biol Chem 2015;290:310-24.

11. Selvanathan SP, Graham GT, Grego AR, et al. EWS-FLI1 modulated alternative splicing of ARID1A reveals novel oncogenic function through the BAF complex. Nucleic Acids Res 2019;47:9619-36.

12. Tsai YS, Dominguez D, Gomez SM, et al. Transcriptomewide identification and study of cancer-specific splicing events across multiple tumors. Oncotarget 2015;6:6825-39.

13. Hong W, Zhang W, Guan R, et al. Genome-wide profling of prognosis-related alternative splicing signatures in sarcoma. Ann Transl Med 2019;7:557.

14. Yang X, Huang WT, He RQ, et al. Determining the prognostic significance of alternative splicing events in soft tissue sarcoma using data from The Cancer Genome Atlas. J Transl Med 2019;17:283.

15. Kim LC, Song L, Haura EB. Src kinases as therapeutic targets for cancer. Nat Rev Clin Oncol 2009;6:587-95.

16. Shukla GC, Singh J. Mutations of RNA splicing factors in hematological malignancies. Cancer Lett 2017;409:1-8.

17. Das S, Anczukow O, Akerman M, et al. Oncogenic splicing factor SRSF1 is a critical transcriptional target of MYC. Cell Rep 2012;1:110-7.

18. Huelga SC, Vu AQ, Arnold JD, et al. Integrative genomewide analysis reveals cooperative regulation of alternative splicing by hnRNP proteins. Cell Rep 2012;1:167-78.

19. David CJ, Chen M, Assanah M, et al. HnRNP proteins controlled by c-Myc deregulate pyruvate kinase mRNA splicing in cancer. Nature 2010;463:364-8.

20. Cancer Genome Atlas Research Network. Comprehensive and Integrated Genomic Characterization of Adult Soft Tissue Sarcomas. Cell 2017;171:950-65.e28.

Cite this article as: Ren X, Jiang Q, Li L. Survival-associated alternative splicing signatures in soft tissue sarcomas. Ann Transl Med 2020;8(5):149. doi: 10.21037/atm.2020.01.113 